\title{
Effect algebras, witness pairs and observables
}

\author{
Gejza Jenča * \\ Department of Mathematics and Descriptive Geometry \\ Faculty of Civil Engineering \\ Slovak Technical University \\ Radlinského 11 \\ Bratislava 81368 \\ Slovak Republic \\ gejza.jenca@stuba.sk
}

\begin{abstract}
The category of effect algebras is the Eilenberg-Moore category for the monad arising from the free-forgetful adjunction between categories of bounded posets and orthomodular posets.

In the category of effect algebras, an observable is a morphism whose domain is a Boolean algebra. The characterization of subsets of ranges of observables is an open problem. For an interval effect algebra E, a witness pair for a subset of $\mathrm{S}$ is an object living within E that "witnesses existence" of an observable whose range includes S. We prove that there is an adjunction between the poset of all witness pairs of $\mathrm{E}$ and the category of all partially inverted E-valued observables.
\end{abstract}

\section{Introduction and motivation}

Let $E$ be an effect algebra. We say that a subset $S$ of $E$ is coexistent if and only if there is a Boolean algebra $B$ and a morphism of effect algebras $\phi: B \rightarrow E$ (an observable) such that $S$ is a subset of the range of $\phi$.

From the point of view of physics, the notion of coexistence is well motivated by its application in mathematical foundations of quantum mechanics, see for example [14 and [3]. From the purely mathematical point of view, one can hope that the study of coexistent subsets can shed at least some light at the enigmatic structure of general effect algebras.

To deal with the notion of coexistence, in [9, 10] we introduced and studied a new notion called witness mapping. For a subset $S$ of an interval effect algebra $E$ in a partially ordered abelian group $G$, a witness mapping for $S$ is a mapping from the finite subsets of $S$ to $E$ satisfying certain conditions.

\section{Effect algebras, observables and witness mappings}

\subsection{Effect algebras}

An effect algebra [5, 13, 7] is a partial algebra $(E ; \oplus, 0,1)$ with a binary partial operation $\oplus$ and two nullary operations 0,1 satisfying the following conditions.

(E1) If $a \oplus b$ is defined, then $b \oplus a$ is defined and $a \oplus b=b \oplus a$.

(E2) If $a \oplus b$ and $(a \oplus b) \oplus c$ are defined, then $b \oplus c$ and $a \oplus(b \oplus c)$ are defined and $(a \oplus b) \oplus c=$ $a \oplus(b \oplus c)$.

${ }^{*}$ This research is supported by grants VEGA G-1/0297/11,G-2/0059/12 of MŠ SR, Slovakia and by the Slovak Research and Development Agency under the contracts APVV-0073-10 and APVV-0178-11.

N. Galatos, A. Kurz, C. Tsinakis (eds.), TACL 2013 (EPiC Series, vol. 25), pp. $109-112$ 
(E3) For every $a \in E$ there is a unique $a^{\prime} \in E$ such that $a \oplus a^{\prime}$ exists and $a \oplus a^{\prime}=1$.

(E4) If $a \oplus 1$ is defined, then $a=0$.

Every effect algebra is a poset under a partial order given by $a \leq b$ iff $(\exists c) a=b \oplus c$.

The class of effect algebras can be considered a common superclass of several important classes of algebras: orthomodular lattices and posets [11, 2], orthoalgebras [6, MV-algebras [4, 16. In particular, every Boolean algebra is an effect algebra.

\subsection{Observables and coexistent subsets}

Let $E, F$ be effect algebras. A mapping $\phi: E \rightarrow F$ is a morphism of effect algebras if and only if the following conditions are satisfied:

(EM1) $\phi(1)=1$.

(EM2) If $a, b \in E, a \perp b$ then $\phi(a) \perp \phi(b)$ and $\phi(a \oplus b)=\phi(a) \oplus \phi(b)$.

Definition 1. We say that a subset $S$ of an effect algebra is coexistent if there exists a Boolean algebra $B$ and an observable $\alpha: B \rightarrow E$ such that $S \subseteq \alpha(B)$.

\subsection{Interval effect algebras}

Let $(G, \leq)$ be a partially ordered abelian group and $u \in G$ be a positive element. For $0 \leq a, b \leq$ $u$, define $a \oplus b$ if and only if $a+b \leq u$ and put $a \oplus b=a+b$. With such a partial operation $\oplus$, the closed interval $[0, u]_{G}=\{x \in G: 0 \leq x \leq u\}$ becomes an effect algebra $\left([0, u]_{G}, \oplus, 0, u\right)$. Effect algebras which arise from partially ordered abelian groups in this way are called interval effect algebras, see [1].

Let $\mathbb{H}$ be a Hilbert space, let $\mathcal{B}_{s a}(\mathbb{H})$ be the set of all bounded self-adjoint operators on $\mathbb{H}$. For $A, B \in \mathcal{B}_{s a}(\mathbb{H})$, write $A \leq B$ if and only if, for all $x \in \mathbb{H},\langle A x, x\rangle \leq\langle B x, x\rangle$. Then $\left(\mathcal{B}_{\text {sa }}(\mathbb{H}),+, 0\right)$ is a partially ordered abelian group. The identity operator $I$ is a positive element of this group.

The prototype interval effect algebra is the standard effect algebra $\mathcal{E}(\mathbb{H})=[0, I]_{\mathcal{B}_{s a}(\mathbb{H})}$. $\mathcal{E}(\mathbb{H})$ plays an important role in the unsharp observable approach to the foundations of quantum mechanics, see for example [3].

\subsection{Witness mappings}

Let $E$ be an interval effect algebra in a partially ordered abelian group $G$. Let $S \subseteq E$. Let us write $\operatorname{Fin}(S)$ for the set of all finite subsets of $S$. We write $I(\operatorname{Fin}(S))$ for the set of all pairs of comparable elements of the poset $(\operatorname{Fin}(S), \subseteq)$, that means, $I(\operatorname{Fin}(S))=\{(X, Y) \in$ $\operatorname{Fin}(S) \times \operatorname{Fin}(S): X \subseteq Y\}$.

For every mapping $\beta: \operatorname{Fin}(S) \rightarrow G$, we define a mapping $D_{\beta}: I(\operatorname{Fin}(S)) \rightarrow G$. For $(X, A) \in I(\operatorname{Fin}(S))$, the value $D_{\beta}(X, A) \in G$ is given by the rule

$$
D_{\beta}(X, A):=\sum_{X \subseteq Z \subseteq A}(-1)^{|X|+|Z|} \beta(Z) .
$$

The transform $\beta \mapsto D_{\beta}$ is (essentially) a Möbius inversion with respect to the poset $(\operatorname{Fin}(S), \subseteq)$; see [9] for details. In [9], we introduced and studied the following notion:

Definition 2. Let $E$ be an interval effect algebra, $S \subseteq E$. We say that a mapping $\beta: \operatorname{Fin}(S) \rightarrow$ $E$ is a witness mapping for $S$ if and only if the following conditions are satisfied. 
(A1) $\beta(\emptyset)=1$,

(A2) for all $c \in S, \beta(\{c\})=c$,

(A3) for all $(X, A) \in I(\operatorname{Fin}(S)), D_{\beta}(X, A) \geq 0$.

For every MV-algebra $M$, the mapping $\Lambda: \operatorname{Fin}(M) \rightarrow M$ is a witness mapping (Corollary 2 of $[9]$ ).

Let $\mathbb{H}$ be a Hilbert space, let $S$ be a pairwise commuting subset of $\mathcal{E}(\mathbb{H})$. The mapping $\Pi: \operatorname{Fin}(S) \rightarrow \mathcal{E}(\mathbb{H})$ given by $\Pi\left(\left\{x_{1}, \ldots, x_{n}\right\}\right)=x_{1} \ldots x_{n}$. is a witness mapping (Proposition 9 of $[9]$ ).

\section{New results}

\subsection{Where do the effect algebras come from}

It was proved by Kalmbach in 12 that every bounded lattice $L$ can be embedded into an orthomodular lattice $K(L)$. However, $K$ is not a functor from the category of lattices to the category of orthomodular lattices. On the positive side, as proved in [15, the Kalmbach construction $K(\cdot)$ can be extended to all bounded posets; for a bounded poset $P, K(P)$ is then an orthomodular poset (OMP). Moreover, as proved in $[\underline{8}, K$ is right adjoint to the forgetful functor from the category of OMPs to the category of bounded posets. Thus, we obtain a monad on the category of bounded posets, which we call the Kalmbach monad.

Theorem 1. The category of effect algebras is isomorphic to the Eilenberg-Moore category for the Kalmbach monad.

This way, the category of effect algebras arises from the category of orthomodular posets.

\subsection{Witness pairs and partially inverted observables}

So far, the most important result concerning witness mappings is the following theorem.

Theorem 2. ([9], Theorem 3) Let $E$ be an interval effect algebra. $S \subseteq E$ admits a witness mapping if and only if $S$ is coexistent.

We show that this theorem is a consequence on the fact that a pair of functors is adjoint.

Let $E$ be an interval effect algebra, let $S \subseteq E$ and let $\beta$ be a witness mapping for $S$. We call the pair $(\beta, S)$ a witness pair in $E$. Suppose that there is another witness pair $\left(\beta^{+}, S^{+}\right)$ such that $S^{+} \supseteq S$ and $\beta^{+}$restricted to $\operatorname{Fin}(S)$ is equal to $\beta$. We then say that $\left(\beta^{+}, S^{+}\right)$extends $(\beta, S)$, in symbols $\left(\beta^{+}, S^{+}\right) \sqsupseteq(\beta, S)$. It is easy to see that $\sqsupseteq$ is a partial order on the set of all witness pairs. We denote the corresponding poset by $\boldsymbol{W} \boldsymbol{i t}(E)$. This is one of our categories.

The other category is the category of partially inverted E-valued observables, denoted by $\boldsymbol{O b s}^{*}(E)$. The objects of this category are all quadruples $\left(B, S, f, f^{*}\right)$, where

- $B$ is a Boolean algebra,

- $S$ is a subset of $E$,

- $f: B \rightarrow E$ is an observable,

- $f^{*}: S \rightarrow B$ is a right inverse of $f$ on $S$, that means, $f \circ f^{*}=\operatorname{id}_{S}$.

An arrow $p:\left(B, S, f, f^{*}\right) \rightarrow\left(B^{\prime}, S^{\prime}, g, g^{*}\right)$ is a morphism of Boolean algebras $p: B \rightarrow B^{\prime}$ such that 
- $g \circ p=f$,

- $S \subseteq S^{\prime}$,

- for every $X \in \operatorname{Fin}(S) \bigwedge p\left(f^{*}(X)\right)=\bigwedge g^{*}(X)$.

Let us define a functor $G: \boldsymbol{O b s}^{*}(E) \rightarrow \boldsymbol{W} \boldsymbol{i t}(E)$ : on objects, we define $G\left(B, S, f, f^{*}\right)=$ $(\beta, S)$, where $\beta: \operatorname{Fin}(S) \rightarrow E$ is given by $\beta(X)=f\left(\bigwedge f^{*}(X)\right)$. Looking at the proof of Proposition 6 of [9], we see that $(\beta, S)$ is a witness pair. The arrows in $\boldsymbol{O b s}^{*}(E)$ were defined so that $G$ is a functor. The result can now be formulated as follows:

Theorem 3. G is a left adjoint.

Theorem 2 is now an obvious corollary of Theorem 3

Let us denote the right adjoint of $G$ by $F$. It turns out that $F(\beta, S)=\left(F_{B}(S), S, \alpha_{S}, \eta_{S}\right)$, where $F_{B}(S)$ is the Boolean algebra freely generated by $S, \alpha_{S}$ was previously defined in 9$] \eta_{S}$ is the unit of the standard free-forgetful adjunction between Boolean algebras and sets.

\section{References}

[1] M.K. Bennett and D.J. Foulis. Interval and scale effect algebras. Advances in Applied Mathematics, 19:200-215, 1997.

[2] L. Beran. Orthomodular Lattices, Algebraic Approach. Kluwer, Dordrecht, 1985.

[3] P. Busch, P. Lahti, and P. Mittelstaedt. The Quantum Theory of Measurement. Springer Verlag, 2nd edition, 1996.

[4] C.C. Chang. Algebraic analysis of many-valued logics. Trans. Amer. Math. Soc., 88:467-490, 1959.

[5] D.J. Foulis and M.K. Bennett. Effect algebras and unsharp quantum logics. Found. Phys., 24:13251346, 1994.

[6] D.J. Foulis and C.H. Randall. Operational quantum statistics. I. Basic concepts. J. Math. Phys., 13:1667-1675, 1972.

[7] R. Giuntini and H. Greuling. Toward a formal language for unsharp properties. Found. Phys., 19:931-945, 1989.

[8] John Harding. Remarks on concrete orthomodular lattices. International Journal of Theoretical Physics, 43(10):2149-2168, 2004.

[9] G. Jenča. Coexistence in interval effect algebras. Proc. Amer. Math. Soc., 139:331-344, 2011.

[10] Gejza Jenča. Extensions of witness mappings. Order, 29(3):533-544, 2012.

[11] G. Kalmbach. Orthomodular Lattices. Academic Press, New York, 1983.

[12] Gudrun Kalmbach. Orthomodular lattices do not satisfy any special lattice equation. Archiv der Mathematik, 28(1):7-8, 1977.

[13] F. Kôpka and F. Chovanec. D-posets. Math. Slovaca, 44:21-34, 1994.

[14] G. Ludwig. Foundations of Quantum Mechanics. Springer-Verlag, Berlin, 1983.

[15] René Mayet and Mirko Navara. Classes of logics representable as kernels of measures. Contributions to General Algebra, 9:241-248, 1995.

[16] D. Mundici. Interpretation of AF $C^{*}$-algebras in Lukasziewicz sentential calculus. J. Functional Analysis, 65:15-53, 1986. 